\title{
Models of machines and models of phenomena
}

\author{
SUSAN G. STERRETT \\ Department of Philosophy, Duke University
}

\begin{abstract}
Experimental engineering models have been used both to model general phenomena, such as the onset of turbulence in fluid flow, and to predict the performance of machines of particular size and configuration in particular contexts.
\end{abstract}

Various sorts of knowledge are involved in the method -- logical consistency, general scientific principles, laws of specific sciences, and experience. I critically examine three different accounts of the foundations of the method of experimental engineering models (scale models), and examine how theory, practice, experience are involved in employing the method to obtain practical results.

Models of machines and mechanisms can be (and generally are) involved in establishing criteria for similar phenomena, which provide guidance in using events to model other events. Conversely, models of phenomena such as events that model other events can be (and generally are) involved in experimentation on models of machines. 
I conclude that often it is not more detailed models or the more precise equations they engender that leads to better understanding, but rather an insightful use of knowledge at hand to determine which similarity principles are appropriate in allowing us to infer what we do not know from what we are able to observe.

In December 2003, a pilot set out to fly a full-scale replica of a machine that had been flown a hundred years earlier. The flight was planned as part of a centennial celebration of the first sustained human-piloted, powered, controlled heavier-than-air flight. The attempt, made December 17th, took place at the same spot -- Kill Devil Hills, North Carolina -- where Orville Wright made a successful, historically significant flight with his flying machine, and exactly one hundred years to the minute after it. The organization that built the replica had spent years building an exact replica of the structure and the engine, using exactly the same materials used in the original, even using period tools to build some of its parts.

But the flight did not go as hoped. The replica of the flyer flown in December 1903 did not get off the ground on that day in 2003 when the re-enactment was attempted in front of the crowd of over 30,000 people who had come to witness it.

CBS News reported the event with the clever headline: "Wright Re-enactment Goes Wrong." (Associated Press, 2003) This evaluation of the event as a failure, however, was only an evaluation of that particular event as a reenactment of the event on December 17, 1903. That is, put as a descriptive statement, the headline was simply stating the lack of similarity in performance between two particular situations: the one on December 17th 1903 and the one on December 17th 2003. 
The organization sponsoring the event drew a different comparison than the headline CBS News used would imply:

Our attempt at flight on December 17, 2003 replicated almost exactly the Wright brothers' first public attempt at flight, May 23, 1904. Lack of wind, engine trouble, and Wilbur got as far as the end of the rail...and went nowhere. (Wright Experience, 2004)

Thus, as a re-enactment of a different event that took place on May 23, 1904, they claimed, the event was actually a success! What occurred on December 17, 2003 at Kill Devil Hills, they pointed out, was in fact similar to another particular event -- not the one that took place exactly a hundred years previous to it in that same location, but to an event that occurred in 1904, in a field called Huffman Prairie in Ohio. Actually, the CBS headline notwithstanding, the body of the CBS news article text conceded their claim: "Though disappointing, the failed first try at a re-enactment was not historically inaccurate." (Associated Press, 2003).

The claim had some merit: despite the painstaking attention to detail expended in constructing their model of the machine -- an exact, full-scale replica, in this case -- what we're often most interested in, and what constitutes physical similarity, is similar performance. That is, the goal is to have the phenomena of interest associated with the thing being modelled arise in the model, too -- here, the phenomenon of interest is that the flying machine become airborne in a controlled, manned, sustained flight. The replication of details such as the exact day of the year, time of day, and geographical location are not relevant to bringing about that phenomenon. The atmospheric conditions are.

There are other phenomenon associated with the original event in 1903 that are not captured by any possible re-enactment of it in 2003 . Nobody would claim that any re-enactment of the 1903 event, no matter how successful, would carry 
with it the historical significance of the original event. The original event was historically significant in that it proved that the problem of controlling sustained powered human-piloted flying machines had finally been solved. The later effects this achievement would have on society as a whole --- how, once the news spread to Europe, money would be invested by nations as well as by private industrialists in airplanes and airplane manufacturing ventures, that there would be major changes in how fast mail could be delivered, in how warfare would be conducted, and so on --- are not associated with the re-enactment. Yet, this is not because economic and social phenomena cannot be the basis of similarity between particular situations.

In fact, judgements of similarity regarding the historical significance of the 1903 event have been made, just recently. I am referring, of course, to the historical flights of the privately developed spacecraft SpaceShipOne, which has been referred to as the "Wright Flyer of spacecraft" (Shostak, 2004) The similarity to Orville Wright's historic flight is being drawn on the basis of the historical significance of the event, rather than on the basis of similarity of the physics of the event. SpaceShipOne won a competition with a 10 million dollar prize, called the X-Prize, which required repeated, controlled space flights by a spacecraft whose design and execution was privately funded. Several self-made entrepreneurs dedicated time and significant parts of their personal fortunes towards the design of a spacecraft built to win the prize, with the ultimate goal of a successful commercial venture of a new sort -- suborbital space travel.

Those who call the spacecraft "the Wright Flyer of spacecraft" do so because they think it will change the nature of space travel in society. Like the Wright Flyer, it was privately developed and cost a fraction of the sum spent by a government-funded program with the same goal. Comparisons were made between the fact that the Wright Brothers constructed their own wind tunnel, and the fact that the SpaceShipOne inventor likewise improvised in lieu of using 
expensive government or university research facilities: for example, by using the back of a pickup truck speeding in a straight line through the desert as a test rig for studying airflow over airfoils. Like the Wright Flyer, SpaceShipOne will provide a flight experience hitherto unavailable that many are eager to pay for, at a price they can actually pay. In drawing a comparison between Orville Wright's historic flight in December of 1903 and the flights being flown in pursuit of the XPrize in October 2004, the similarity between the particular event in 1903 and the particular event in 2004 is based on the economic and societal significance of the event. Such comparisons are relevant to answering questions about what kinds of resources and incentives are necessary conditions for certain kinds of technological achievements.

Now, as I've just stated it, what constitutes similarity is relative to a phenomenon -- the phenomenon of interest. The notion of similarity I invoke here is the notion of similarity used in physics in the most general sense: physical similarity, of which some special kinds are dynamic similarity, kinematic similarity, and hydrodynamic similarity. The general idea is that the mapping between things -events, machines, systems, phenomena --- preserves the structure of the situation that is relevant to the phenomenon of interest. When two systems are physically similar, a similarity mapping can be drawn based on keeping certain relationships (or ratios) of the quantities or elements in one system the same as certain relations (or ratios) of the quantities or elements in the other. Having the same relevant relations (or ratios) between elements or quantities is what guarantees or shows that the two systems have the same structure. Now, each event, machine, system, or phenomena has lots of different kinds of structures; these are picked out or highlighted by the relationships (dimensionless ratios) that characterize that particular structure. How do you know what structure to identify? Which relationships (or ratios) are kept the same between model and thing modeled is a matter of what structure is of interest, and that in turn depends on what phenomenon is of interest. Different structures are responsible (in some 
cases we might even want to say causally responsible) for different phenomena, and different structures are picked out by different similarity mappings.

I just talked about three different similarity comparisons. First, the static mechanical-structural similarity between the exact replica of the machine produced in 2003 and the original one produced in 1903. Then, the dynamic similarity of the 2003 event and an event in 1904 -- occasions on which an exact replica of the Wright Flyer and the original, respectively, performed similarly poorly. And, finally, there was the similarity between the 1903 event and SpaceShipOne's successful flight in 2004, based upon similarity of historical significance. Thus, similarity is itself defined in terms of interests. If we are talking about human endeavors, these will be interests humans have. That similarity turns out on this account to be relative to human interests is not an oversight on my part --- nor is it a drawback to the use of similarity. It does not mean that similarity is subjective as opposed to objective, merely that similarity is relative to a phenomenon. There is no such thing as absolute similarity; similarity can only be similarity with respect to some feature or phenomenon of interest.

The use of similarity in scientific inference is ubiquitous, although it is not always explicitly recognized. For instance, observations of events in the laboratory are considered informative about other things and events that go beyond the specifics of the observed case. In so doing, we are implicitly assuming there is a class of events or situations that are similar to the given event, and that the given event is informative of other events in that class. This is true in general, whether the area is mechanics, acoustics, hydrodynamics, chemistry, or whatever. A twofold question now arises: (i) when an observation is made on a specific setup in a controlled laboratory setting or in a natural setting, what determines the class of other events to which it is deemed similar? And (ii) what kind of correspondence is there between the observed event and these other events? 
To take an especially striking example in the philosophical literature, consider experiments done using laboratory setups in hydrodynamics. Margaret Morrison has discussed Ludwig Prandtl's experimental investigations of fluid flow over a sphere using a water tank. Let's take a closer look at what is involved in the step she describes as follows: "Although the existence of frictional forces were known, what the experiments indicated was that the flow about a solid body can be divided into two parts, the thin layer in the neighbourhood of the body where friction plays an essential role (the boundary layer) and the region outside this layer where it can be neglected." ((Morrison, 1999, p. 55) The question I want to ask about this is: "What was the basis for making inferences about cases other than the specific case in the laboratory setup Prandtl used?" That is, what was the criterion for saying which other cases are like the one observed, and in what respect? What is our basis for being able to say which other objects and setups would behave like the one observed, were the experiment run on them? Think of the disappointing re-enactment of the flight attempted with an exact replica of the airplane I described at the opening of this paper. It is not that the explanation of the performance is a mystery to us; the point is that that example illustrates that distinctions need to be made in order to pick out the cases that will behave similarly from those that will not. In his paper in this volume, Michael Heidelberger discusses Ludwig Prandtl's work on the theory of the boundary layer.

In general, if we want to play it absolutely safe in drawing inferences from an observed case, we would have to restrict our inference to cases using exactly the same fluid at exactly the same temperature and pressure, flowing at exactly the same velocity, in exactly the same geometrical configuration, at exactly the same scale. We would also have to use exactly the same size and shape of object, made of exactly the same material with exactly the same surface finish. Even after all that, we'd have to hope that no external time-varying factors such as sunspots made a difference to the phenomena we'd observe. Of course this is 
unsatisfactory. The observations are taken to have more generality, and they need to be, if we are to gain any practical knowledge. But, on what basis is the generalization of the observation made?

The experimenter needs to have enough insight into what the phenomena depends upon to know how to characterize equivalent classes of situations, that is, situations that are equivalent with respect to giving rise to the phenomenon of interest. Although some insights can be gained by varying individual variables such as velocity, density, viscosity, size, and so on one by one, ultimately what is needed, however it is obtained, is the ability to characterize situations and determine which situations are similar to each other with respect to the phenomenon of interest. Without that crucial ability, all one has is an inventory of observations of individual cases.

Thus, an experimenter needs to have some criterion for similarity, and, in fact, is employing similarity criteria whether or not he or she realizes it. That's my general point, and my point about Prandtl's experiment in particular is that Prandtl did not start from scratch with respect to criteria for hydrodynamical similarity. To carry out the kind of investigations he did, he needed to be able already to characterize the kind of situations he was going to investigate, and that means he used some criteria of hydrodynamical similarity, whether good or bad. A researcher using incorrect similarity criteria is not going to get robust results from his or her investigations. Prandtl used appropriate similarity criteria, and he obtained spectacularly robust results.

Fortunately, Prandtl already had pretty good reasons for suspecting that one of the nondimensional factors on which the phenomenon he was investigating would depend would be the nondimensional parameter now known as Reynolds number. The Reynolds number is a product involving fluid density, relative fluid velocity, a linear dimension, and viscosity, all in a single nondimensional 
parameter. This had been the result of many years of work by Osborne Reynolds, beginning with his observation that temperature seemed to affect fluid flow phenomena, and in turn that viscosity varies with temperature. From Reynolds' work, too, Prandtl -- and all the world --- knew that the formation of patterns of vortices in viscous fluids was an important phenomenon to investigate and categorize. And, again, the relevant criterion of similarity of situations -- i.e., the basis on which to identify equivalence classes of situations, was the Reynolds number.

Another clue about similarity that Prandtl had to work with came from William Froude's work on towing ship hulls in water tanks. Froude had shown that when a ship hull was moving with respect to a fluid, there was a layer of water that formed near the ship hull, and Froude carrried out investigations specifically on the causes and effects of this "skin" phenomenon (which would later be called the boundary layer). Froude's case itself illustrates my general point.

Several decades before Prandtl's experiments, Froude used a water tank for the purpose of experimenting on scaled-down models of ship hulls being towed through water. Now, the question is, what did experimental observations on that case tell him? The answer is found in the similarity these experimental setups have to other setups. Since similarity is relative to a phenomenon, the question only makes sense in the context of specifying a phenomenon of interest. Using the similarity law of his day, Froude said that observations on his model told him the resistance of a ship hull of the same shape but of a size $n$ times as large, travelling at that velocity times the square root of $\mathrm{n}$, if you scale the resistance up by $n 3$. This basis for similarity is similarity with respect to the effects of gravitational forces, and it only gave good results if viscosity was not important to the phenomenon of interest, the overall resistance the ship experienced in travelling through the water. Froude did realize, though, that as you used smaller and smaller sized scale models, there were disproportionate effects that was 
probably due to the viscosity of the fluid. Froude observed that viscous shear on the full size ship hull models created a layer of fluid that "stuck" against the hull, but further hypothesized that the fluid within this layer was in motion with respect to the ship hull. Once he began to suspect that in fact the phenomenon of interest, resistance to a ship travelling in the water, might depend not only on gravitational forces but also on viscous forces, he devised a method to account for it. (Rouse and Ince, 1957, p. 185) The details here are not important. The point is that the observations on the laboratory model do not stand on their own -in order to associate the observations made in one situation with any other situation, you need a basis on which to make similarity judgements.

Froude's investigations led him to attribute this difference to the phenomenon now called the boundary layer, and in turn to attribute that phenomenon to fluid friction, or viscosity. This was another clue about what was a relevant criteria for similarity. All this was the background knowledge about hydrodynamical similarity that preceded Prandtl's work. This background knowledge enabled him to properly characterize the fluid flow situations he was investigating. My point is that this was crucial in two ways: it was crucial to his being able to achieve the experimental results he did in the first place, and it was crucial to the results he obtained being applicable to other situations.

Let me bring out this same two-sided point with another example from history of science: the principle of corresponding states, from chemistry and thermodynamics. This principle, due to van der Waals, has appeared a few times in discussions by philosophers of science (Glymour, 1970; Morrison 1988 ), but usually in the contexts of scientific realism or theory reduction. The limitations of Boyle's law (PV = RT) were well-known in van der Waals's day: Boyle's law applied only for low pressures and high temperatures, it didn't apply to the liquid state, and it didn't describe the transition between vapor and liquid states. The popularity of steam engines led to a practical interest in vapor-liquid 
transitions, and to observations of rapidly increasing pressures of enclosed vapor at temperatures above the boiling point of water. Experiments were also carried out on enclosed liquids and vapors of other substances, which led to cataloguing the critical temperatures of various liquids, and to liquefying gases at ambient temperatures by compressing them. (Sengers, 2002, p. 14)

What may have stimulated Van der Waals to develop his equation was Thomas Andrews's work on the state of a substance at which vapor and liquid are identical, which Andrews called the "critical point". Andrews also investigated what happened when compressing vapor above the critical temperature, coining the term "continuity of states." (Sengers, 2002, p. 14 ) Van der Waals added two parameters to Boyle's Law, "a" and "b", both constants meant to reflect characteristics of a specific substance. The two parameters were to account for the factors of the volume the molecules occupy and the mutually attractive forces between them. So, what van der Waals did here was to use some partial knowledge about the model he suspected was responsible for the phenomenon being described.

The Van der Waals equation was far more than a detailed version of Boyle's law -- unlike Boyle's law, it explained fluid criticality and continuity of states, and it qualitatively described empirical results of new investigations being carried out. But the most significant conceptual step was the principle of corresponding states. The principle of corresponding states arises out of applying the van der Waals equation to find the critical points of a given substance in terms of the parameters $a$ and $b$, and then, essentially, using the values of critical pressure, critical volume, and critical temperature of the substance as a unit of measure. The pressure expressed as a ratio of pressure to critical pressure is called a "reduced pressure", and reduced temperature and reduced volume are similarly defined. If we retain the expressions for the critical values in terms of $a$ and $b$, and express pressure, temperature, and volume in these units of measure, we 
obtain a form of the van der Waals equation in which the postulated constants a and $b$ no longer appear. The resulting equation is then in terms of reduced temperature, reduced pressure, and reduced volume. Since it does not contain the substance-specific constants $a$ and $b$, it applies to all substances. The principle was thus an improvement over the van der Waals equation, as knowledge of the critical values of a substance obviated the need to determine the substance-specific parameters a and b. Van der Waals did come to recognize that even the principle of corresponding states was only approximate. Yet, as one textbook puts it: "This is a truly remarkable result." pointing out that the equation expressing the principle of corresponding states "is universal: all characteristics of individual fluids have disappeared from it or, rather, have been hidden in the reduction factors." (Sengers, 2002, p. 25)

What I want to emphasize about the principle of corresponding states here is that we can think of it as providing a similarity criterion. It puts into one equivalence class the states of all substances that are at the same reduced pressure, temperature, and volume. This aspect of the principle provoked another scientist, Heike Kamerlingh Onnes, to look for its basis in another, known similarity principle. In his Nobel lecture, Onnes said that the law of corresponding states "had a particular attraction for me because I thought to find the basis for it in the stationary mechanical similarity of substances and from this point of view the study of deviations in substances of simple chemical structure with low critical temperatures seemed particularly important." (Onnes, 1913) He won the Nobel prize in 1913 for his success in applying these ideas to the problem of liquefying helium.

As with most similarity principles, there are two complementary faces to the law of corresponding states: on the one hand it supplies criteria for characterizing situations, and on the other it provides a means of translating the specific features of one situation into the corresponding specific features of another. As 
for the first aspect of the principle -- characterizing situations -- the characterization of a state is in terms of reduced pressure, temperature, and volume and is independent of the substance. As for the second aspect, it is easy to see how the translation between situations works here: take, for example, pressure. Suppose you conclude you have a substance in a certain state; then you know the reduced pressure. The pressure for a particular substance in that state can be found by multiplying the reduced pressure by the critical pressure of that substance. (Sengers, 2002, p. 25)

I have been emphasizing the importance of such similarity principles as background knowledge necessary for carrying out investigations, even laboratory investigations. Let me close by saying a little about the source of such similarity principles.

In both hydrodynamic similarity and the law of corresponding states, the similarity principle is a consequence of an equation deemed applicable to the phenomenon being investigated: hydrodynamic similarity is a consequence of a fluid flow equation, and the law of corresponding states is a consequence of the van der Waals equation. In general, if you've got a governing equation describing a phenomenon available, a similarity principle can be obtained straightforwardly by manipulating the equation into a form such that the requisite nondimensional parameters are obtained by inspection of the equation. However, you don't need a governing equation in order to obtain a similarity principle; you can get by with much less.

Both hydrodynamical similarity and the law of corresponding states can be derived from merely the knowledge of the list of quantities upon which the phenomenon depends. That means that you need only know the form of the equation in the most general sense, i.e., that the phenomenon is a function of quantity 1 , quantity 2 , etc. As long as the list is complete, and doesn't contain 
extraneous quantities, the method of dimensional analysis will show a valid similarity principle valid; i.e., that the Reynolds number is indeed a basis for hydrodynamical similarity, and that the law of corresponding states is indeed a basis for thermodynamical similarity. Further, even if you don't have a candidate similarity principle to verify, the method will also always yield results, in the form of one or more nondimensional parameters that will provide a basis for similarity. It will not yield an equation describing the phenomenon, but no such equation is needed in order to establish similarity. There is one drawback to the method; since it is based upon a purely logical analysis of the dimensions of the quantities involved, the parameters produced by the method might not have physical significance. So, the complaint is often made that the method is not of much practical use.

There is a third approach that tries to make use of all the information and physical insight one might have at hand. I am taking the notion, called "configurational analysis", from a field of research called fire modelling, but it is applicable to any field. Now, fires are not tidy things -- all kinds of factors matter, such as the configuration of the space where the fire occurs, how the configuration is changed by the fire devouring parts of a building, the kinds of materials and atmospheric conditions it encounters as it progresses, and so on. The phenomena to be modelled have probably not even been exhaustively catalogued. Various types of pure science are brought to bear on the problem, although this is not a situation where we reasonably expect to obtain equations that describe the resulting phenomena. We may use our speculations or knowledge about physical mechanisms or fundamental principles to pick out important factors that affect the phenomenon of interest. What we use this information for, however, is not to solve equations. Rather, the goal is to figure out similarity conditions. We then use the similarity conditions to build models and, after using the model experimentally, to infer corresponding particular features of the situations they are meant to model. Alternatively, we can use 
observational data in lieu of experimental data; then, the similarity criteria help in categorizing and making inferences from cases at hand.

Now, as I said, one drawback of dimensional analysis was that the nondimensional parameters the process produces are not guaranteed to have any physical significance, and in order to experiment with a model, we need to have parameters to vary that have some physical significance. What the configurational analysis approach involves is first using physical insight in conjunction with the logical principle of dimensional homogeneity. In the words of the author of a paper entitled "Fire Modeling" from which the notion of "configurational analysis" comes, the method "permits the welding of similitude theory and a 'feeling' for the problem at hand". The guiding idea for employing a 'feeling' for the problem is explained as follows:

Consider a device or process subject to change in space or time. Quantitative statements about the system will be statements about force, matter, or energy. A statement of the existence of a balance of forces, for example, will of necessity contain terms which are dimensionally identical, and division through by one of the terms will produce a set of force ratios. These ratios must be the same in similar systems. Similar statements may be made about conservation of matter and energy. (Hottel, 1961, p. 32) 1

Thus, very general principles such as conservation laws are employed to guide the selection of nondimensional parameters on which a similarity principle for the phenomenon of interest can be obtained. As Hottel has stated things, the starting point is to identify the "device or process", but I think it clear that this includes identifying the phenomena associated with the device or the process that we are interested in, and any that might be causally important to it. This is where background knowledge such as that small viscosities can be important, or 
that the mutual attraction between the molecules of an enclosed gas can be important, is brought into the development of the similarity conditions.

Hottel mentions ratios of forces, masses, and energy, but it is not hard to see that the same approach could be taken for problems involving quantities of other sorts as well. Becker (1976) takes a generalist approach along such lines, then spells out what the relevant ratios are for applications in various disciplines. The key parts of this approach are to identify sources, fluxes, and boundaries, and then apply insights about continuity and conservation to them. We can extend the approach beyond the physical sciences to the social and behavioral sciences as well. Economists are used to drawing analogies between fluid flows and flows of currency. Even Freud used hydraulic and thermodynamic analogies. It is easy to see that the general point about using flow balances could be employed whenever a process or device is conceived in terms of fluxes of any sort -- flows of information, birth and death rates, flows of capital investment, flows of goods and natural resources, of technological expertise, flows of nerve energies, flows of inhibitory signals, and so on.

My point at the beginning of the paper was that similarity between particular situations was based upon similarity with respect to a phenomenon of interest. When the equations describing a phenomenon are already in hand, there is more than enough information to establish similarity criteria. However, similarity criteria can be established with less information as well. There are methods that produce similarity criteria that do not require specifying the device or process, but the similarity criteria they produce may not be in a meaningful or useful form. Now we see how a rather abstract characterization of a device or process -- a model of the device or process, we might well call it -- is employed in obtaining similarity criteria of practical significance that can be useful in designing experiments or collecting observational data. 
Summarizing these reflections, we see that there are several ways that models can be involved in applying science:

-- Anytime observations are used to make generalizations or inferences beyond an observed case, one event is being used as a model of another event, or of an equivalence class of other events. These comparisons are based upon similarity criteria, and are relative to a phenomenon or phenomena of interest. Even characterizing experiments and observations involves employing similarity criteria, whether the person doing so realizes it or not. Hence this use of a model -- using one event as a model of another, which is a model in the sense of modelling phenomena -- is ubiquitious in all sorts of scientific activities.

-- If the similarity criteria is obtained from an equation describing the phenomena, a model in the more traditional sense of providing a mechanism may be involved inasmuch as a model of the device or process is often used in deriving the equation. (However, having such an equation is not necessary; similarity criteria can be obtained even when an equation describing the phenomena of interest is not known.)

-- In order to obtain physically meaningful and hence practically useful similarity criteria without the use of a governing equation, a characterization of the device or process in terms of equilibrium or steady-state flow fluxes, sources, and boundaries is valuable in conjunction with the formal method of dimensional analysis. Balances and conservation principles can be applied to guide the construction of the nondimensional parameters used as similarity criteria.

Thus, models of machines and mechanisms can be (and generally are) involved in establishing criteria for similar phenomena, and these criteria provide guidance in using events to model other events. Conversely, models of 
phenomena such as events that model other events can be (and generally are) involved in experimentation on models of machines.

I like Margaret Morrison's remark, situating my points here in general philosophy of science; she says: "A good deal of literature in philosophy of science has emphasized the role of analogies in inductive inference; what I take Sterrett to have shown is why, in certain respects, analogies work." (Morrison, this volume, p. xx ) On the account I have presented here, we see that often it is not more detailed models or the more precise equations they engender that leads to better understanding. Often, what is most helpful is an insightful use of the knowledge at hand to determine which similarity principles are most appropriate in allowing us to infer what we do not know from what we are able to observe. I hope my discussion here helps in getting more precise about that insight.

Acknowledgements: I am totally indebted to the work of Henry Becker on scale modelling in chemical engineering research for drawing attention to Hottel's paper, which Becker cites for an explanation of what he calls "configurational analysis". Becker's treatment (Becker, 1976) is reflective and philosophical. 


\section{References}

ASSOCIATED PRESS (2003) Wright re-enactment goes wrong. Appeared on CBS News website.

http://www.cbsnews.com/stories/2003/08/21/tech/main569635.shtml accessed January 30, 2004.

BECKER, H. (1976) Dimensionless Parameters: Theory and Methodology. (London, Applied Science).

GLYMOUR, C. (1970) On some patterns of reduction, Philosophy of Science, 37 , pp. $340-353$.

HOTTEL, H.C. (1961) Fire modeling, in: BERL, W. G. (ed.) The Use of Models in Fire Research. (Washington, D.C., National Academy of Sciences - National Research Council, Publication 786).

MORRISON, M. (1988) Reduction and realism, PSA1988, Volume 1, pp. 286293.

(1999) Models as autonomous agents, in MORGAN, M. S. \& MORRISON, M. (Eds) Models as Mediators (Cambridge, Cambridge University Press), pp. $38-65$.

ONNES, HEIKE KAMERLINGH (1913) Investigations into the properties of substances at low temperatures, which have led, amongst other things, to the preparation of liquid helium, Nobel Lecture, December 11, 1913.

http://nobelprize.org/physics/laureates/1913/onnes-lecture.pdf, accessed October 11, 2004. 
ROUSE, H. \& INCE, S. (1957) History of Hydraulics. (lowa City, Institute of Hydraulic Research)

SENGERS, J. L. (2002) How fluids unmix: discoveries by the school of Van der Waals and Kamerlingh Onnes (Amsterdam, Koninklijke Nederlandse Akademie van Wetenschappen).

SHOSTAK, SETH (2004) Where does 'outer space' begin? MSNBC News Archive http://msnbc.msn.com/id/5287945/, accessed January 30, 2005.

WRIGHT EXPERIENCE (2004) The Wright experience in flight. Online resource http://www.wrightexperience.com/edu/12_17_03/html/121703.htm, accessed September 22, 2004. 
Biographical Note

Susan G. Sterrett joined the department of Philosophy at Duke University in Durham, North Carolina, USA in Fall 2000 after earning a Ph.D. in Philosophy from the University of Pittsburgh. She is especially interested in analogical reasoning and model-based reasoning. A preprint of another of her papers on experimental models, "Physical Models and Fundamental Laws: Using One Piece of the World to Tell About Another", is available on the Philosophy of Science Association's archives (at http://philsci-archive.pitt.edu/archive 100000720). Correspondence: Department of Philosophy, Box 90743, 201 West Duke Building, Durham, NC 27708. Email: sterrett@duke.edu 